\section{Focal electroencephalography rhythm asymmetry due to focal skull fibrous dysplasia}

\author{
Michael A. Meyer \\ Department of Neurology, Sisters \\ Hospital, Buffalo, NY, USA
}

\begin{abstract}
An unusual pervasive and persistent asymmetry in background rhythm was found on surface electroencephalography (EEG) recordings in a 22 year old with new onset of generalized seizure activity. Radiographic correlation with computed tomography, positron emission tomography and bone scan imaging uncovered that the higher amplitude left frontal-parietal background activity was related to a circumscribed area of left frontalparietal fibrous dysplasia affecting the skull. This case report emphasizes that the presumed higher electrical conductance of fibrous dysplasia lead to a greater transparency of normal background rhythms, and must be taken into account as a form of breach rhythm for accurate EEG interpretation
\end{abstract}

\section{Case Report}

A 22 year old female with new onset of generalized seizure activity of unknown cause underwent a head computed tomography (CT) examination that revealed focal hypo-density within the left frontal bone, consistent with fibrous dysplasia and extended back into the left parietal bone as well (Figure 1). Despite anti-convulsant medication, additional nocturnal generalized seizure events recurred, prompting a whole body bone scan that was negative except for focally elevated $99 \mathrm{mTc}$ MDP uptake in the left frontal-parietal region (Figure 1). Positron emission tomography (PET) imaging failed to reveal any focal metabolic defect within the brain (Figure 1) and displayed a slight diffuse prominence to FDG uptake within the left frontal-parietal bone. Magnetic resonance imaging of the brain also failed to reveal any significant abnormalities but did also show the area of fibrous dysplasia to have mildly prominent $\mathrm{T} 2$ signal as seen on the T2 weighted axial section (Figure 1).

Electroencephalography (EEG) examination revealed a persistent asymmetry in activity in the left frontal leads, with activity from this region being of higher voltage and better defined (Figure 1) than right hemispheric activity (Figure 1). No definite epileptogenic discharges were revealed but did suggest episodic left frontal sharp wave activity compared to the right, but in retrospect, this appears to be of an artifactual nature due to the reduced resistance to current flow across the area of fibrous dysplasia, which apparently conducts EEG potentials with less impedance.

\section{Discussion}

Fibrous dysplasia is often a benign incidental disorder where normal bone is replaced by fibrous connective tissue and can be at one location (monostotic) or less frequently seen at multiple sites on bone scan (polyostotic). Easily identified on bone scan as an area of focally increased tracer uptake within CT hypodense areas, the lesion often has low levels of metabolic activity on PET scan, but exceptions have been reported.1,2 Other PET tracers can better characterize the metabolic profile of the lesion through the use of 11C-methioinine or
Correspondence: Michael Andrew Meyer, Department of Neurology, Sisters Hospital, 2157 Main Street, Buffalo, NY 14214, USA.

Tel.: + 1.716.862.2750.

E-mail: michaelandrewmeyer@gmail.com

Key words: seizure, electroencephalography, skull, fibrous dysplasia, computed tomography.

Conflict of interests: the author declares no potential conflict of interests.

Received for publication: 11 February 2014.

Accepted for publication: 4 April 2014.

This work is licensed under a Creative Commons Attribution NonCommercial 3.0 License (CC BYNC 3.0).

CCopyright M.A. Meyer, 2014

Licensee PAGEPress, Italy

Neurology International 2014; 6:5347

doi:10.4081/ni.2014.5347

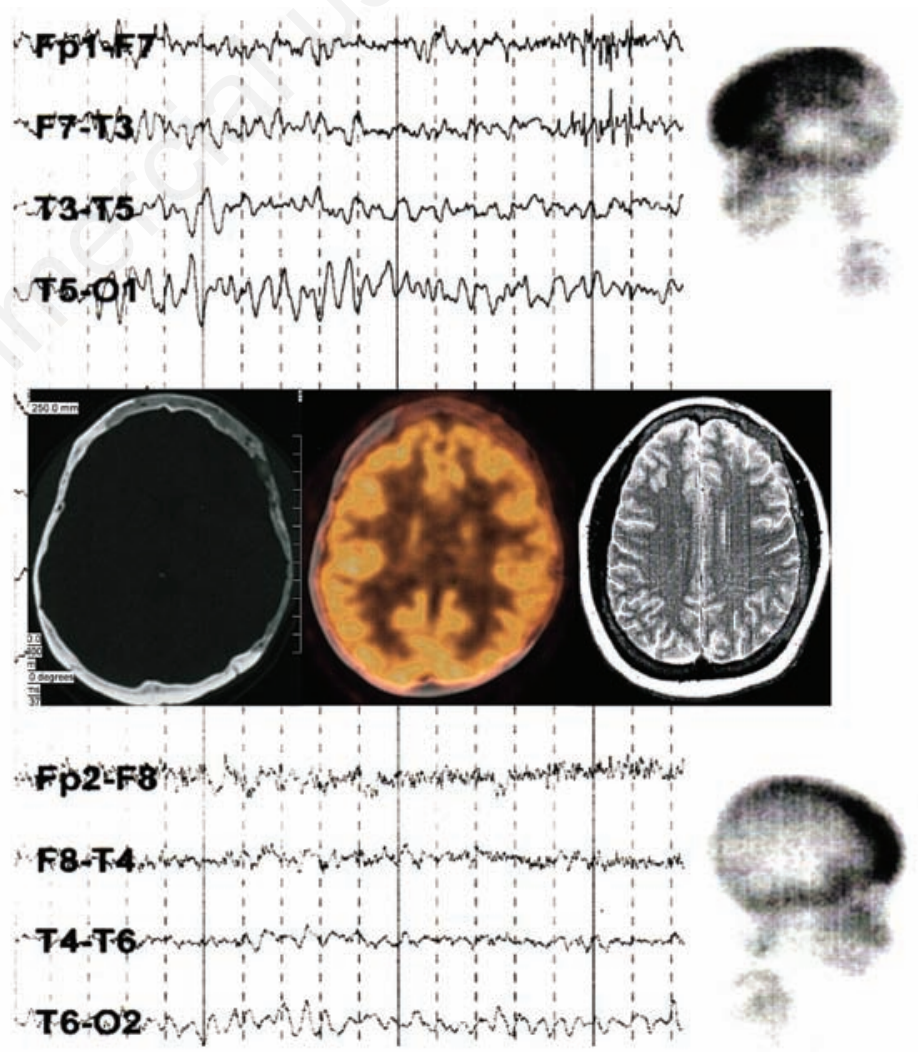

Figure 1. Composite of left hemispheric electroencephalography (EEG) activity at top, versus right hemispheric EEG activity from the same time epoch shown at bottom. Image at left of middle panel reveals head computed tomography (CT) windowed to show only bone densities, with middle color image illustrating PET/CT of the brain; right hand portion of middle panel shows axial T2 weighted section at same level as PET with mildly increased T2 signal within the left frontal-parietal focus of fibrous dysplasia (FD). Left lateral planar image of $99 \mathrm{mTc}$ MDP bone scan at top right reveals characteristically intense uptake in the left frontal-parietal region suggesting FD as the diagnosis, which stands in contrast to the right lateral view of the skull (right lower image). 
11C-choline.3,4 In only 4 to $10 \%$ of cases does malignant transformation occur into other forms such as osteosarcoma or fibrosarcoma. ${ }^{3}$

A small number of case reports on the coexistence of fibrous dysplasia with Epilepsy are noted with some of these reports suggesting a causative inter-relationship, ${ }^{5-8}$ which clearly seemed not be the case for the patient discussed here, and seemed to be incidental. However, the important unique finding of this case, not previously reported elsewhere, is recognition that misleading EEG interpretations can occur if the electrical conductance properties of focal fibrous dysplasia affecting the skull is not accounted for. In this case, an apparent asymmetry in waveform amplitude and definition appeared from the left frontal leads, similar to the well known breach rhythm phenomenon that occurs over post-surgical skull defects. ${ }^{9}$ Although a breach rhythm effect has not been previously reported with fibrous dysplasia, a case of something similar has been reported to due a focal skull defect from multiple myeloma. ${ }^{10}$

\section{Conclusions}

In summary, monostotic fibrous dysplasia of the skull can be found on CT and /or brain magnetic resonance imaging and needs to be taken into account when interpreting EEG activity recorded over the involved region, as diminished resistivity to current flow is likely and produces an apparent asymmetry in waveform definition and amplitude, with the underlying activity being easier to record and more apparent. In conclusion, focal EGG asymmetries related to focal fibrous dysplasia of the skull represent a form of breach rhythm with regards to its electrographic classification.

\section{References}

1. Su MG, Tian R, Fan QP, et al. Recognition of fibrous dysplasia of bone mimicking skeletal metastasis on 18F-FDG PET/CT imaging. Skeletal Radiol 2011;40:295-302.

2. Aras M, Ones T, Dane F, et al. False positive FDG PET/CT resulting from fibrous dysplasia of the bone in the work-up of a patient with bladder cancer: case report and review of the literature. Iran $\mathbf{J}$ Radiol 2012;10:41-4.

3. Tsuyuguchi N, Ohata $\mathrm{K}$, Morino $\mathrm{M}$, et al. Magnetic resonance imaging and [11C]methyl-L-methionine positron emission tomography of fibrous dysplasia-two case reports. Neurol Med Chir (Tokyo) 2002;42:341-5.

4. Gu CN, Hunt CH, Lehman VT, et al. Benign fibrous dysplasia on $[(11) \mathrm{C}]$ choline PET: a potential mimicker of disease in patients with biochemical recurrence of prostate cancer. Ann Nucl Med 2012;26:599-602.

5. Kanda M, Yuhki I, Murakami Y, et al. Monostotic fronto-orbital fibrous dysplasia with convulsion-case report. Neurol Med Chir (Tokyo) 2002;42:36-9.

6. Soler R, Iváñez V, Muñoz-Torrero JJ, Barreiro P. [Epileptic seizures as the first sign of fibrous bone dysplasia]. Rev Neurol 1999;28:976-8. [Article in Spanish].

7. Nishioka K, Masuda Y, Inokuchi I, et al. A case of monostotic fibrous dysplasia of the temporal bone associated with epileptic seizure. Acta Med Okayama 1982;36:453-62.

8. Bertoni JM, Kooi KA. Fibrous dysplasia of the skull, with seizures and focal electroencephalographic findings. Dev Med Child Neurol 1978;20:483-7.

9. Brigo F, Cicero R, Fiaschi A, Bongiovanni LG. The breach rhythm. Clin Neurophysiol 2011;122:2116-20.

10. van Doorn J, Cherian PJ. Neurological picture. Breach rhythm related to a solitary skull lesion caused by multiple myeloma. J Neurol Neurosurg Psychiatry 2008;79:819. 